\title{
Towards Controlled Transmission: A Novel Power-Based Sparsity-Aware and Energy-Efficient Clustering for Underwater Sensor Networks in Marine Transport Safety
}

\author{
Israa Adil Hayder ${ }^{1, *(\mathbb{D}}$, Sundas Naqeeb Khan ${ }^{2}$, Faisal Althobiani ${ }^{3}$, Muhammad Irfan ${ }^{4, *} *^{\mathbb{D}}$, Muhammad Idrees ${ }^{5}$, \\ Saeed Ullah ${ }^{6}$, Faisal Alsaaq ${ }^{3}$, Adam Glowacz ${ }^{7, *} \mathbb{1}$, Iwona Goldasz ${ }^{8}$, Marcin Tomczyk ${ }^{9}$ and Radu Martis ${ }^{10}$
}

1 Department of Computer Science, COMSATS University Islamabad, Islamabad 44000, Pakistan

2 Faculty of Computer Science and Information Technology, Universiti Tun Hussein Onn Malaysia, Parit Raja, Batu Pahat, Johor 86400, Malaysia; sndskhan87@gmail.com

3 Faculty of Maritime Studies, King Abdulaziz University, Jeddah 21589, Saudi Arabia; falthobiani@kau.edu.sa (F.A.); falsaaq@kau.edu.sa (F.A.)

4 Electrical Engineering Department, College of Engineering, Najran University Saudi Arabia, Najran 61441, Saudi Arabia

5 Department of Computer Science and Engineering, University of Engineering and Technology Lahore, Narowal 0542, Pakistan; midrees10@uet.edu.pk

6 Department of Computer Science, Federal Urdu University of Science and Technology, Islamabad, Karachi 75300, Pakistan; saeedullah@gmail.com

Citation: Hayder, I.A.; Khan, S.N.; Althobiani, F.; Irfan, M.; Idrees, M.; Ullah, S.; Alsaaq, F.; Glowacz, A.; Goldasz, I.; Tomczyk, M.; et al. Towards Controlled Transmission: A Novel Power-Based Sparsity-Aware and Energy-Efficient Clustering for Underwater Sensor Networks in Marine Transport Safety. Electronics 2021, 10, 854. https://doi.org/ 10.3390 /electronics10070854

Academic Editor: Juan-Carlos Cano

Received: 30 January 2021

Accepted: 1 April 2021

Published: 3 April 2021

Publisher's Note: MDPI stays neutral with regard to jurisdictional claims in published maps and institutional affiliations.

Copyright: (c) 2021 by the authors. Licensee MDPI, Basel, Switzerland. This article is an open access article distributed under the terms and conditions of the Creative Commons Attribution (CC BY) license (https:// creativecommons.org/licenses/by/ $4.0 /)$.
7 Department of Automatic Control and Robotics, Faculty of Electrical Engineering, Automatics, Computer Science and Biomedical Engineering, AGH University of Science and Technology, al. A. Mickiewicza 30, 30-059 Kraków, Poland

8 Faculty of Environmental and Power Engineering, Cracow University of Technology, Warszawska 24 Str., 31-155 Cracow, Poland; iwona.goldasz@pk.edu.pl

9 Faculty of Electrical and Computer Engineering, Cracow University of Technology, Warszawska 24 Str., 31-155 Cracow, Poland; marcin.tomczyk@pk.edu.pl

10 Department of Electrical Machines, Marketing and Management, Universitatea Tehnica Cluj-Napoca I UT Cluj, 400114 Cluj, Romania; radu.martis@gmail.com

* Correspondence: adil_israa@yahoo.com (I.A.H.); miditta@nu.edu.sa (M.I.); adglow@agh.edu.pl (A.G.)

\begin{abstract}
Energy-efficient management and highly reliable communication and transmission mechanisms are major issues in Underwater Wireless Sensor Networks (UWSN) due to the limited battery power of UWSN nodes within an harsh underwater environment. In this paper, we integrate the three main techniques that have been used for managing Transmission Power-based Sparsity-conscious Energy-Efficient Clustering (CTP-SEEC) in UWSNs. These incorporate the adaptive power control mechanism that converts to a suitable Transmission Power Level (TPL), and deploys collaboration mobile sinks or Autonomous Underwater Vehicles (AUVs) to gather information locally to achieve energy and data management efficiency (Security) in the WSN. The proposed protocol is rigorously evaluated through extensive simulations and is validated by comparing it with state-of-the-art UWSN protocols. The simulation results are based on the static environmental condition, which shows that the proposed protocol performs well in terms of network lifetime, packet delivery, and throughput.
\end{abstract}

Keywords: marine transport; safety; underwater wireless sensor networks; routing protocols; energy consumption; controlled transmit power; autonomous underwater vehicle; void hole; reliable data delivery

\section{Introduction}

Seventy percent of the world is covered by water. Therefore, for human beings, underwater environments are quite important. Moving further, underwater resources are paid attention by scientists due to the depletion of terrestrial resources. UWSNs have many uses, such as being commonly used in coastal surveillance and assurance, an expectation of sea calamity, observation of underwater contamination, military protection, 
road assistance, management of the aquatic oceanic climate, investigation of underwater properties, monitoring of water quality, and so on in [1-3].

Protocols designed for Terrestrial Wireless Sensor Networks (TWSN) are unable to perform efficient data transmission in the aquatic world [4]. As a result, there are differences between TWSNs and UWSNs from different perspectives, such as the use of acoustic connections rather than radio links. UWSNS topology is more difficult than TWSNs because underwater nodes travel with water streams unreservedly and adapt their positions constantly, it is more difficult to locate nodes in UWSNs than TWSNs, and node placement is often sparse [5]. Moreover, UWSNs face other challenges such as low bandwidth resulting in low underwater data rate, low propagation speed resulting in high delay. Moreover, underwater acoustic waves propagate at a speed of $1500 \mathrm{~m} / \mathrm{s}$, which is 5 orders less than radio waves, and propagation occurs over multiple paths that leads to signal distortion through a fading propagation environment [6]. Nodes underwater also face high mobility resulting in network topology instability [7].

Sensor nodes used with acoustic transmitters and receivers are placed underwater. In water, these sensor nodes may be either mobile or static. Furthermore, data is sent to surface sink nodes by the sensor nodes, often using lower depth sensors as relay stations. Autonomous Underwater Vehicles (AUVs), on the other hand, will collect data from sensors immediately. AUV routing necessitates a great deal of planning. In [8], a tracking method based on the cloud-like model data association algorithm is introduced to track underwater various targets. In comparison, the use of AUVs can incur additional costs. Throughout the UWSN system, routing protocols play a vital role. These protocols are responsible for producing suitable routes and data on those routes through sensor nodes to stations where significant data can be gathered. These protocols must be robust to extreme aquatic conditions to achieve maximum performance. These protocols, for instance, can successfully solve energy limitations, mobility of nodes, high SINR, high-end delays, etc. Following a detailed analysis of the latest UWSN routing schemes and some critical findings on the problem of the energy void, the decreased propagation ratio of the packet, and the overall load on sensor nodes closer to the sink are made. The closing remarks are as follows:

- Most of the existing routing schemes use maximum transmission power of sensor nodes while transmitting packets to neighboring nodes or sinks. This process ends with the rapid absorption of energy by sensor nodes. In comparison, the high transmitting capacity use often produces more channel interference [9]. Consequently, the probability of energy hole creation is also increased.

- Unbalanced transmission data load distribution resulted in a rapid decline in energy nodes and created network energy gaps. Owing to the energy hole occurrence, various sensor nodes die before the rest. This energy hole problem results in consuming high energy and decreases the lifetime of the network. Extra loads on nodes closer to the sink node can be removed by the existing methods. The nearest nodes to the sink consume their resources quickly due to the heavy load, while nodes farther away consume their resources slowly. Consuming energy rapidly also decreases the Packet Delivery Ratio (PDR) [10].

- Most of the existing UWSN considers the usage of one or multiple static sinks at the water surface for data collection. The sensor nodes' data is reached towards the nearest sinks by using various routing mechanisms. This approach poses great problems in terms of scalability when the network size is increased. Moreover, it creates a bottleneck and lowers the performance of the network. Additionally, the UWSN closer to these sinks depletes their energy very rapidly and creates energy holes that force the rest of the nodes for distant communication and result in rapid energy depletion. Hence, the overall network lifetime is reduced.

The proposed protocol addresses the above-mentioned issues by integrating three approaches, including clustering, mobile sinks use, and transmit power control. The challenges that we encountered during the design were as follows: 
The first challenge is how to manage and use mobility in the networks to continuous connectivity of the nodes and AUV for improving communication efficiently.

The second challenge is hole energy creation to some extent, regarding the highly heterogeneous sensors, and the vast diversity of energy and depth of the sensors to apply clustering technique.

The third challenge is the cost of the transmission. The energy cost is still a critical apprehension in the sensor system.

The fourth challenge is how to exploit the AUVs, which are high-cost devices, with the collaborated $\mathrm{CHs}$ node selection, to address transmission distance challenge for achieving the stabilization of networks in sparse regions. The main contributions of the proposed scheme are three-fold:

- $\quad$ Propose a novel adaptive power control routing protocol to efficiently use their energy and to reduce the channel interference that ultimately increases the network lifetime, reduces the interference, and avoids the energy hole creation.

- Furthermore, the clustering technique is incorporated in the dense regions to reduce the duplication of transmitted packets and to balance the load of data packets that potentially lowers the interference and improves the battery usage of sensor nodes.

- Finally, local deployment of mobile sinks or AUVs to retrieve packets through two $\mathrm{CHs}$ as a relay that successfully received data packets from the source to collaborative protocol using a predefined distance threshold to evaluate AUV position-aware mobility such that the distance between transmitting and receiving devices is calculated. If the distance is less than or equal to the distance threshold value, then the data packet is transmitted and received at the receiver, otherwise the data packet is dropped, and that distance threshold value depends on the RSSI value calculated by the receiver. The protocol is designed for one-way data transmission from mobile sinks to the static sink for enhancing the network lifetime by reducing transmission distance and by minimizing the interference. Moreover, it also helps to avoid energy holes and increases successful packet delivery.

The rest of this article is structured as follows: the relevant work done in UWSNs is checked and classified in Section 2. The method of the schemes proposed is described in Section 3. Section 4 deals with the simulation setup and performance evaluation of the proposed scheme, followed by Section 5's conclusion.

\section{Related Work}

In this section, some pertinent research on routing protocols in UWSNs is presented, along with their capabilities, benefits, and disadvantages. We also grouped the routing protocols into protocols that are localization-conscious, localization-free and cluster-based.

\subsection{Localization-Free Routing Protocols}

The authors in [11] show a routing protocol that proficiently handles complex networks. Moreover, local knowledge is required to allow the depth of transfer packets greedily in the direction of the sink. In this paper, the proposed protocol for each qualifying node forwards priority-based packets. Priority is determined by the depth of forwarder nodes that have never sent the same packets together before. The DBR has better network life and a high PDR. However, this greedy method forces the immutable collection of forwarder nodes by which network nodes are partitioned and services are still underused due to being expensive and consuming exorbitant energy [12].

In [13], a routing protocol is proposed where they used two mobile sink nodes to effectively collect the data. Moreover, a metric called "Mobile Sink Efficiency Ratio (MUR)" was presented to quantify the use of the mobile sink node during data collection. This system provided successful results for network lifespan, loss of packets, throughput, and the new MUR metric. However, a high end-to-end delay resulted from this system. The authors suggested a scheme to address the current shortcomings of the schemes in [14] so that they concentrated on the consistency of the connection and routing path problem. 
Connection Efficiency Indication (LQI), Signal-to-Noise Ratio (SNR), and Packet Reception Probability (PRP), an on-site triangular metric routing combination, is used to effectively select the next forwarder, resulting in low routing costs, minimal energy consumption, and increased network life.

The authors suggested "Localization-Free Energy-Efficient Routing (LFEER)" and "Cooperative localization-Free Energy-Efficient Routing (Co-LFEER)" in [15]. First, the residual energy, hops, and failure rate of the link bit at which the data packets were sent were taken into account when selecting a relay. In the latter, the sink receives several data replicas from which it can decide on the data quality. These schemes provided better outcomes for energy optimization and increased PDR. However, as these schemes know the exact positions of sensor nodes, they result in computational complexity and extra use of resources.

\subsection{Localization-Aware Routing Protocols}

In [16], the authors included the use of mobile sensor nodes to reduce the energy hole and keep the sink in its precise position. Clusters are installed on the network for this purpose, and data processing uses mobile nodes. To achieve fuel consumption, smoother network life, and load balancing, this routing approach is intended. The cost of a high end-to-end delay will be compensated by this approach, though.

The writers have suggested a cross-layer reactive routing protocol for [17]. Furthermore, this scheme covered the difficulties described by the standard CARP scheme when the authors did not follow the re-usability estate. Avoiding packet management guarantees longer network life and reduced energy consumption. However, owing to the constant rotating of the nodes, the consequence of this scheme is a decreased throughput and a high loss of course. In [18], the author's aim is to develop an efficient routing algorithm for secure transmission to each of the sinks while also solving the void hole problem. Like DBR, several mobile pontoons are spread on the surface of the water as holding a sink. It has two greedy packet-forwarding mode switches and dealing with a void in communication. However, in DBR, a subset of the upper depth nodes is specified for data-packet forwarding. When a void node is discovered, a detour path for data-packet transmission should be identified. Consequently, knowledge is guided up to lower depths. The gauge used in this way improves the routing performance under continuous node movement conditions. However, it is necessary to measure the node's depth correctly. The PDR has been strengthened, and inefficient performance and heavy energy consumption are its drawbacks. The authors take into account the depth of the existing node, as well as the depth of the next forwarding node in the paper, [19], to reduce the risk of empty holes and resource consumption. The issue of the void hole is not solved by switching packets over two hops, however. The authors of [20] use a technique that adapts the pipeline radius to change the packet-forwarding area to help eliminate redundant packets. This method results in a higher packet delivery ratio, lower energy consumption, and a short end-to-end delay. There is still an issue with the void hole, though.

In [21], authors have developed for asynchronous wireless sensor networks, and calculate the best forwarders based on an estimate of the forwarding score and estimation of residual power. This protocol resolves problems related to load balancing, loss of coverage, and accessibility.

Voltage is provided to pick up a node for the forwarder. However, since the cycling responsibility in this protocol is not as low as 140, asynchronous duty-cycling caused massive delays in waiting times for the next-hop node to wake up. Residual energy chooses a node forwarder. Nevertheless, asynchronous duty-cycling caused the nexthop node to wake up with tremendous delay waiting times as cycling is not as small as 140 in this protocol requirement and the number of forwarders will start waking up simultaneously and result in repetition of packets.

To forward data to the destination node, the authors of [22] suggested a greedy routing scheme. To avoid duplication in GEDAR, nodes that are on lower priority suppress their 
packet transmission. The key function of this technique is that if a void hole occurs, it employs a depth correction scheme, i.e., it moves the node to a new depth in the case of a void node resuming a greedy forwarding. When the void hole is effectively covered, the reliability of the network that uses this device is increased. Moving nodes into new depths, on the other hand, consumes a lot of energy and increases end-to-end latency.

In [23], the authors developed an efficient routing algorithm for safe transmission to each of the sinks as well as solving the void hole problem. The forwarding node is chosen based on the status of the data packet and the cost of the connection. Alternatively, the gauge used needs to perfectly guess the node depth. For regional all-cast routing, the data gathered in-depth may be used. It has increased PDR and the likelihood of void nodes has reduced. Increased oil usage and overhead are the drawbacks. In [24], the author recommended a complex address scheme to assist in choosing an acceptable next-hop neighbor. The suggested approach then assigns hop addresses to each node that helps to forward information. However, this protocol raises the PDR, triggering heavy energy consumption.

\subsection{Cluster-Based Routing Protocols}

In [25], the authors proposed a "cooperative energy-efficient optimal relay selection protocol" for underwater wireless sensor networks. Furthermore, a source node selects a relay node and a sink node on its own, and data is then transferred to the sink node through the relay node. It helps to remove the need for coordination between the source node, relay node, and sink nodes using this technique. Moreover, for receipt or re-transmission of the data, the sink passes the acknowledgment to the source node. The selection of forwarding nodes or relays is based on two parameters: depth and location value. Pressure sensors are used to determine depth, and location is determined by the distance between nodes and the surface. The nearest node to the sink node is chosen as a relay node based on the two values. If the destination node receives a data packet successfully, it sends an acknowledgment to the source node; if the reception is unsuccessful, it requests re-transmission. Data is sent to a relay node after it is chosen, and it relays it to the destination. Therefore, this scheme contributes fewer packet drops to this method. However, the data load on a node's source, relay, and sink increases, which reduces the stability time of the node.

This paper also suggests the FSO and EM energy dissipation model in [26]. To find the optimum cluster collection for Gaussian-distributed UWSNs, the authors looked at the theoretical context. The authors achieved rational results by moving the sink to three additional points, namely the tip, the corners, and the middle of the sensing region. The suggested protocol uses the least amount of energy possible. However, there is a significant end-to-end delay.

Cluster-based sleep-wake scheduling is indicated in [27]. In this paper, the authors employ a strategy in which some of the nodes are presumed to be initiator nodes. These initiator nodes will then choose the CHs. As the head node, the $\mathrm{CH}$ node that is left with high energy is picked. When other nodes are sent to sleep mode, this header node is set to active mode. The propagation is then carried out into the node of the drain by the node of the selected head.

This strategy lowers energy usage, increases the life and distribution ratio of the packets. After all, the $\mathrm{CH}$ total life of the network influences the network's life.

In [28], the authors first performed theoretical analysis to obtain the expected value of nodes in the network. Next, they divided the network into irregular clusters. In this paper, they used the strategy of recovery balance consumption of energy among clusters. In this paper, they have achieved enhanced PDR, consuming less energy, and enhanced network lifetime. However, this scheme results in variation in the network. The suggested particle encoding and fitness functions proposed by the author are used to construct this technique in [29]. The scientists looked at a variety of metrics to improve energy efficiency, including the distance between clusters, the gap between sinks, and the residual energy for cluster nodes. Using these metrics, they were able to derive the weighting function for clusters. 
Scientists reached high PDR values, increased lifetime, and reduced energy consumption in this article. The authors of [30] looked at the trade-off between accuracy and energy efficiency and proposed a solution. In WSNs, the 'adaptive FEC/FWD and FEC/ARQ' clustering mechanisms are based on the channel state and the distance between nodes. We have worked to significantly strengthen our previous work [31] by collaborating with the following organizations:

- The author recommends a complete dfiction class of invalid handling algorithms for UWSNs in three primary classifications based on their literature review: control with power, surpass region-space, and mobility assisted-based strategies. The author recommends a complete fication class of invalid handling algorithms for UWSNs in three primary classifications based on their literature review: control with power, surpass region-space, and mobility assisted-based strategies.

- The author offers an analytical framework for evaluating the performance and tradeoffs of different space-processing algorithms in UWSNs. Considering the features of our model network structure and acoustic interconnections beneath the water, as well as recovery strategies.

- The author carried out numerical tests to assess the overall performance of three widely used methods for designing void-processing algorithms in water sensor networks. The author investigated several network density scenarios in our numerical analysis, which is a key factor influencing the number of empty nodes. According to [32], the geographic routing, routing strategy is hindered by unexpected and dynamic changes in the UWSN topology. Geographic routing strategy is hindered by abrupt and dynamic changes in the UWSN topology, which may result in communication void areas in the network. For example, this routing requires redone-hop neighboring information to send on to the future destination data messages, and it selects the closest neighboring node to the destination to continue forwarding data packets.

This scheme resulted in a more reliable network and more energy efficiency. However, this scheme is effective only for small networks. The proposed scheme addresses the issues pointed out in various above-mentioned UWSN routing schemes and improves network performance by balancing the load distribution, lowering the channel interference, achieving the energy efficiency, avoiding the energy holes in the network by integrating the clustering, mobile sinks use, and transmit power control mechanisms.

Two new methods have been built in [33] to restrict energy consumption and remove the existence of a void zone in [34]. During the first approach, mobile sink nodes or AUVs play an important role in reducing node contractual power consumption, while the second strategy entails planning and cooperation with the first. This improves the efficiency of the proposed cooperative network by transmitting packets along different routes. In the second scheme, RODP increases decrease package leakage and increases transmission delay costs with full power consumption. The first system lowers the use of electricity and keeps the deal running for a long time.

\section{Proposed Methodology}

A single sink network model is assumed in the proposed scheme. As illustrated in Figure 1, it is made up of anchored nodes, relay nodes, and sink nodes. The anchored nodes are located on the seafloor and are primarily used to collect underwater data, while the relay nodes are located at a different underwater location and are responsible for transporting data to the sink and forwarding the collected packets [29]. For the sink node, there are sound and radio modems. For acoustic communication, the former is used, while the latter enables radio communication outside the aquatic environment. The sink node, which is mostly on the water's surface, sends data packets to the satellite, which then sends them to the control center. 


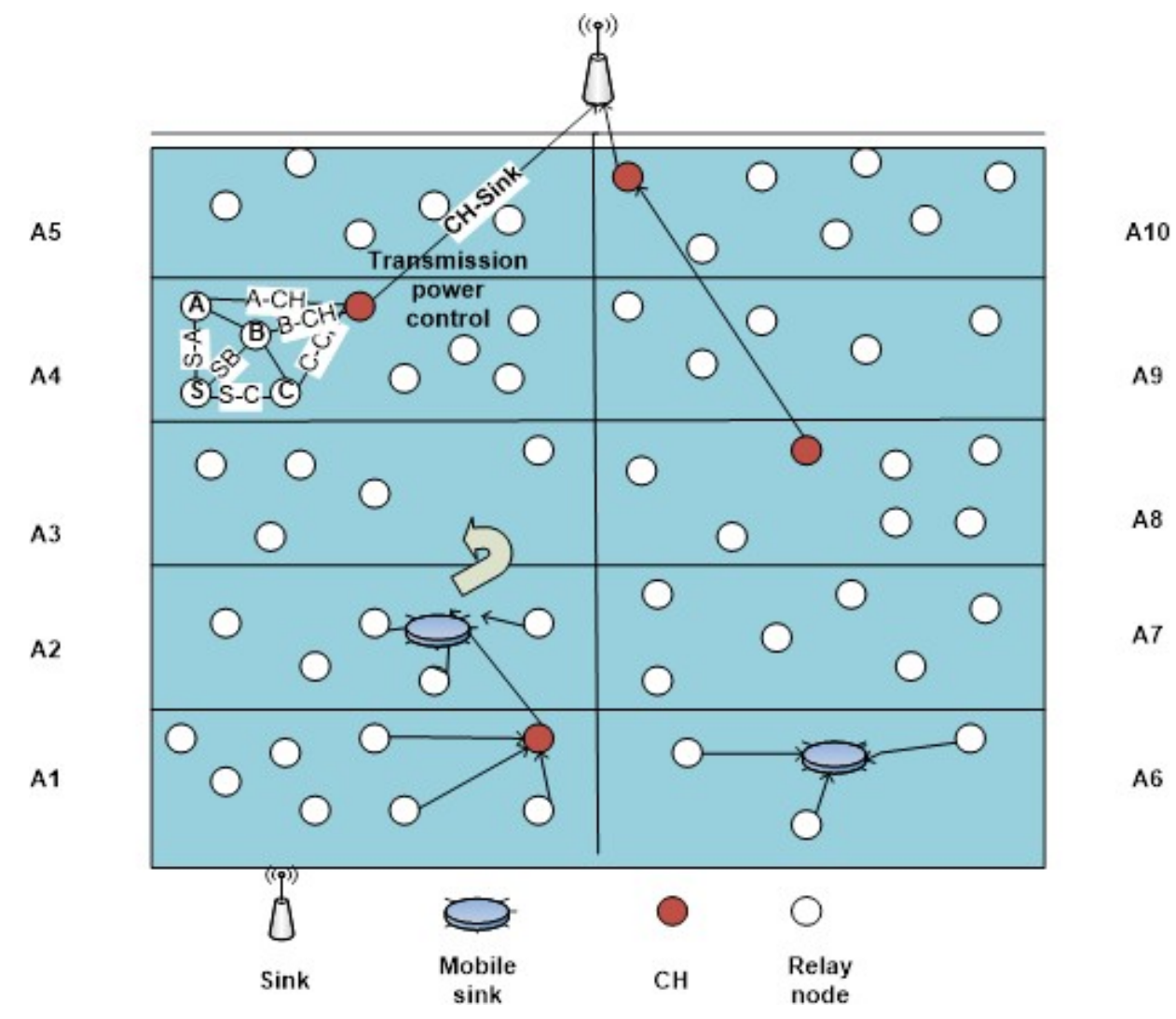

Figure 1. CTP-SEEC System Model.

\subsection{Energy Consumption Model}

The RSSI signal can be distorted due to fading environment, which causes the loss of information. Moreover, it reduces when the distance increases, which indicates the distance. RSSI is disrupted by fading, so it should be measured with channel impairments in mind. As a result, there is a strong need to deal with RSSI when channel impairments are present. The sink node, which is mostly located on the water's surface, sends data packets to the satellite, which then sends them to the control center. Because the signals propagate vertically, the attenuation increases as the distance between them grows. Transmission damage, on the other hand, can be expressed as:

$$
\mathrm{TD}=\mathrm{k}_{\mathrm{TD}} \times 10 \lg \mathrm{r}+\alpha_{\mathrm{TL}} \mathrm{r} \times 10^{-3}
$$

where TD denotes the transmission loss in decibels, and $\mathrm{k}_{\mathrm{TD}}$ denotes the spreading loss in decibels. Furthermore, its value varies depending on the water depth and propagation geometry; in shallow water, $\mathrm{k}_{\mathrm{TD}}$ is kept equal to 1 for cylindrical spreading; in deep water, $\mathrm{k}_{\mathrm{TD}}$ is kept equal to 2 for spherical spreading; The absorption coefficient of the medium, which varies with frequency and is measured in $\mathrm{dB} / \mathrm{km}$, is $\alpha_{\mathrm{TD}}$; $\mathrm{r}$ is the distance between the receiver and the transmitter; The medium's absorption coefficient is measured in $\mathrm{dB} / \mathrm{km}$ for $\mathrm{f}$ in $\mathrm{kHz}$, which can be calculated using the equation:

$$
\alpha_{\mathrm{TD}}(\mathrm{f})=0.11 \frac{\mathrm{f}^{2}}{1+\mathrm{f}^{2}}+44 \frac{\mathrm{f}^{2}}{4100+\mathrm{f}^{2}}+2.75 \times 10^{-4} \mathrm{f}^{2}+0.003
$$

Its frequency is approximately $30 \mathrm{kHz}$. UWSNs have an absorption coefficient of less than $10 \mathrm{~dB} / \mathrm{km}$. The absorption coefficient for chemical absorption in seawater is computed using acoustic frequency, pressure, acidity, temperature, and salinity. The following formula is used to compute the received signal power (RSSi), also known as the received signal power or RSSI:

$$
\mathrm{Y}=\mathrm{Y}+\mathrm{TD}
$$


where epsilon is the returned signal power at the close in-reference distance, and $\mathrm{r}$ is the reference distance. The energy consumption model described in [34] can be used to calculate the minimum transmission power needed at the transmitter to meet power level $C_{0}$ at the receiver end.

$$
C_{t x}=C_{0} \times d^{2} \times 10^{\left(\alpha_{T D}(f) / 10\right)}
$$

The absorption coefficient is $\alpha(f)$, the incoming power is $C_{0}$, the distance between the transmitter and receiver is $\mathrm{d}$, and the received power is $C_{0}$. The energy consumption of wireless sensor networks cannot be applied to UWSNs because the acoustic wave characteristic of an underwater transmission medium varies from that of a radio wave. As a result, the energy consumption model for the underwater acoustic channel is the same as in [34]. The amount of energy required to send $\mathrm{m}$ bits of data over a long distance at a data rate $\mathrm{R}$ is calculated as follows:

$$
\mathrm{E}_{\mathrm{Tx}}(\mathrm{m}, \mathrm{d})=\mathrm{m} \times \mathrm{E}_{\text {elec }}+\frac{\mathrm{m}}{\mathrm{R}} \mathrm{C}_{\mathrm{tx}}
$$

The amount of energy needed to route one bit of data is denoted by $E_{\text {elec }}$. The receiver's radio energy consumption for transmitting $\mathrm{m}$ bits of data is calculated as follows:

$$
\mathrm{E}_{\mathrm{Rx}}(\mathrm{m})=\mathrm{mK}_{\mathrm{r}}
$$

where $\mathrm{K}_{\mathrm{r}}$ is a device-specific constant. The following formula is used to calculate the energy needed to fuse $m$ bits of data:

$$
\mathrm{E}_{\mathrm{DA}}(\mathrm{m})=\mathrm{m} \times \mathrm{E}_{\mathrm{DA} 0}
$$

The device-dependent constant $K_{r}$ is used here. The following formula is used to determine the energy needed to fuse $\mathrm{m}$ bits of data:

\subsection{Adaptive Power Control with Adaptive Clustering Routing Model}

The GRACE [33] principle of gradient cost field establishment, which is inspired by the natural phenomenon of water flowing down a valley from top to bottom, is the foundation of our adaptive power control routing model. Data propagates in the same way, in the direction where it finds the cheapest path. The gradient for the cost field establishment scheme and the factors are described as follows:

- $C_{E, i}$ : The energy cost of the ith node is given by

$$
\mathrm{C}_{\mathrm{E}, \mathrm{i}}=\frac{E_{\mathrm{i}}^{0}}{E_{\mathrm{i}}}
$$

- $\quad C_{L, u} \rightarrow v$ : Link Cost: The cost of a link between a transmitting node $\mathrm{u}$ and a receiving node $\mathrm{v}$ is calculated as follows:

$$
\mathrm{C}_{\mathrm{L}, u \rightarrow \mathrm{v}}=\frac{P_{\mathrm{t}, \mathrm{u}}}{P_{\mathrm{r}, \mathrm{V}}}
$$

where $P_{t, u}$ denotes node u's transmitted power and $P_{r, v}$ denotes node v's received power.

- Route Cost, $C_{N} \rightarrow N$ : Route cost between the path- starting node N1 and the pathterminating node $\mathrm{N} 2$ is given by

$$
\mathrm{C}_{\mathrm{N}_{1} \rightarrow \mathrm{N}_{2}}=\sum_{i=N_{1}}^{N_{2}-1}\left(\Omega_{E} \mathrm{C}_{\mathrm{E}, \mathrm{i}}+\Omega_{L} \mathrm{C}_{\mathrm{L}, \mathrm{i} \rightarrow \mathrm{i}+1}\right)
$$

- The following are some helpful concepts for establishing a cost field: $\Omega_{L}$ Information about the advertising sent from node ' $\mathrm{I}$ ' to its neighbors. 
CI S the cheapest route from node 'I' to sink 'S' This diagram depicts the only low-cost option.

CI JS The routing path from node ' $I$ ' to sink ' $S$ ', using node ' $J$ '. This reflects a single special path originating from node 'I' and ending at Sink ' $\mathrm{S}$ ', out of many possible paths. Where $\Omega_{E}$ and $\Omega_{L}$ are the energy and link cost weighting factors, respectively.

The cost field is defined using the APCEER protocol [9] notion, which is illustrated in Figure 2 where nodes J, K, and L are the Ith node's immediate neighbors.

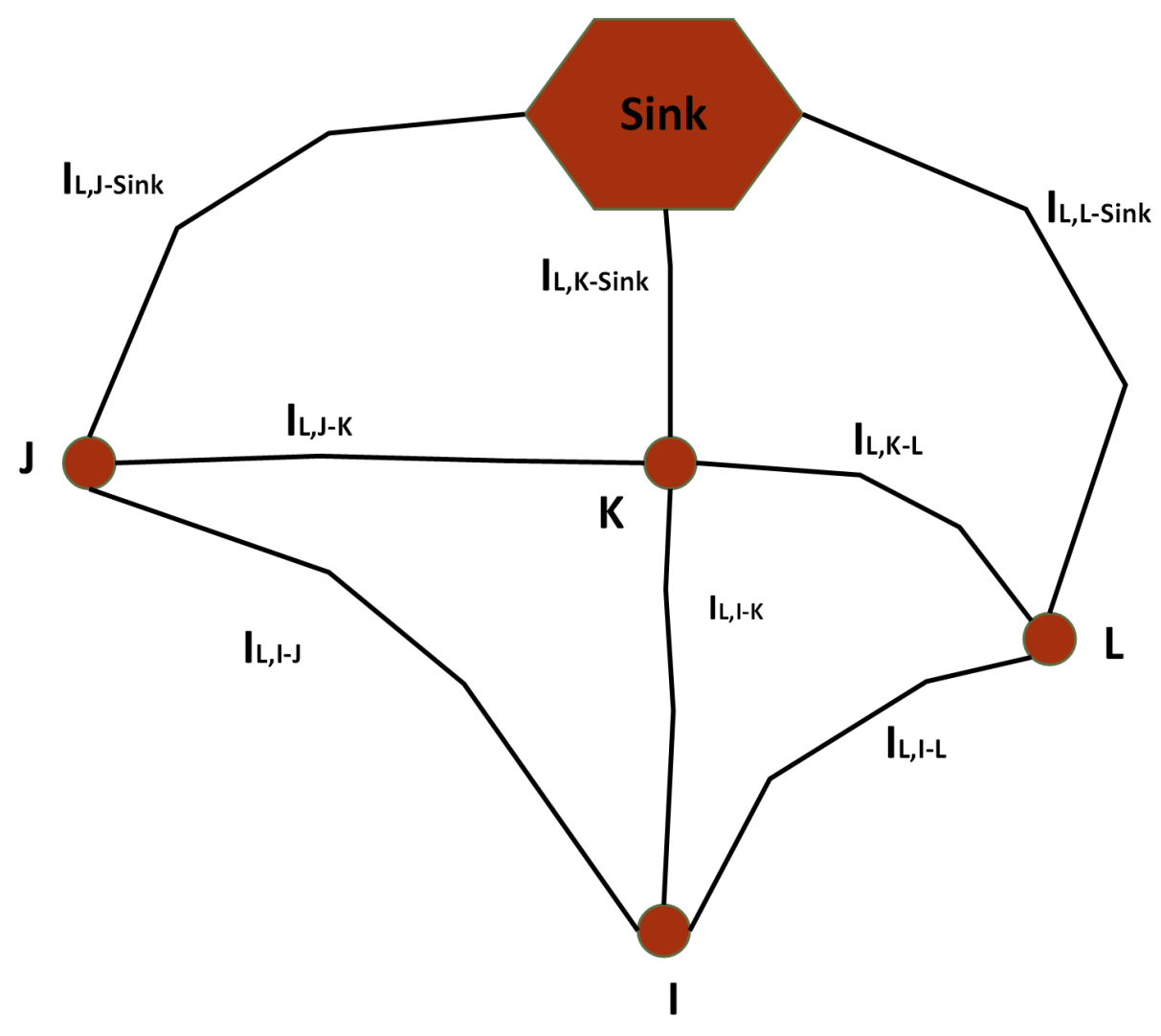

Figure 2. Cost Field Setup.

The cost fields and advertising information of the nodes can be defined as follows:

$$
\begin{aligned}
\alpha_{\mathrm{K}} & =\mathrm{C}_{K \rightarrow S}+\mathrm{C}_{\mathrm{E}, \mathrm{K}} \\
\alpha_{\mathrm{J}} & =\mathrm{C}_{J \rightarrow S}+\mathrm{C}_{\mathrm{E}, \mathrm{J}} \\
\mathrm{C}_{I \rightarrow J \rightarrow S} & =\alpha_{\mathrm{J}}+\mathrm{C}_{L, I \rightarrow J} \\
\alpha_{\mathrm{L}} & =\mathrm{C}_{L \rightarrow S}+\mathrm{C}_{\mathrm{E}, \mathrm{L}} \\
\mathrm{C}_{I \rightarrow L \rightarrow S} & =\alpha_{\mathrm{L}}+\mathrm{C}_{L, I \rightarrow L} \\
\mathrm{C}_{I \rightarrow K \rightarrow S} & =\alpha_{\mathrm{K}}+\mathrm{C}_{L, I \rightarrow K} \\
\mathrm{C}_{I \rightarrow S} & =\min \left(\mathrm{C}_{I \rightarrow J \rightarrow S}, \mathrm{C}_{I \rightarrow K \rightarrow S}, \mathrm{C}_{I \rightarrow L \rightarrow S}\right)
\end{aligned}
$$

Herein, we opted for the concept of adaptive clustering multi-hop routing scheme [34]. Here, the node can be $\mathrm{CH}, \mathrm{CM}$, or $\mathrm{CR}$ in the first, second, third round, respectively. In comparison to its neighbors, each node will choose to become a cluster head based on the selection conditions [32]. To form a cluster, the $\mathrm{CH}$ will relay the "JOIN" message to its neighbors. Otherwise, the node will wait for the "JOIN" message; if it only receives one message, it will become a cluster member. When a node receives a significant number of "JOIN" messages, it selects the sender node with the greatest received power or the shortest distance. 
The cluster formed between nearby nodes, we think, to conserve energy and reduce transmission time. We assume that some nodes will become the CR and forward data to the sink due to the restricted transmission range of the nodes at depths D2 and D3.

If a relay node receives the "JOIN" message from both the upper and lower depth nodes (DL and DL-1), the node decides to become a CR, which acts as a route forwarder. The nodes send a "RESPONSE" message to the $\mathrm{CH}$ to validate the cluster-ID. The CHs at the same level simply send them a "JOIN" message.

Node Si tests Sk's depth if the CH Si receives a "RELAY" message from Sk, and if Sk's depth is near the surface, node $\mathrm{Si}$ or $\mathrm{Dm}(\mathrm{Sk}) \mathrm{Dm}(\mathrm{Si})$, and node $\mathrm{Si}$ adds $\mathrm{Sk}$ as a relay node.

In a cluster scheme, the relay node may gather data from the sensor node or control the network topology. As a result of the node's energy conservation, the nodes' energy consumption has been reduced. Some clustering protocols, on the other hand, have only been developed and studied in two-dimensional (2D) deployments, while UWSN network scenarios necessitate three-dimensional deployments (3D).

\subsection{Network Model}

The network scenario consists of $\mathrm{N}$ dynamic nodes that are deployed in a threedimensional scenario $L \_L \_L$ at random and sparsely. The data source, on the other hand, is the data sensed in the water medium and extracted by the underwater sensor nodes. The sink nodes are placed in the center of the surface in this case.

Because water currents with velocities of around 1-3 m/s move the underwater sensor nodes, the topology changes quickly.

The following is a list of the network's assumptions:

- $\quad$ nNodes should become the cluster head, cluster relay, or cluster member after the first

deployment because they know their own location and the location of the sink node.

- To save energy, the cluster head is rotated between the sensor nodes.

The depth of deployment is split into layers, with each layer identified by the node or sink's transmission range. It can be calculated using the following formula:

$$
n_{\text {de } p t h}=\frac{L}{T x}
$$

where $L$ denotes the network deployment depth and $T x$ denotes the node or sink's transmission range. The network's depth has been grouped into four categories:

- $\quad$ The sink node is designated by D0.

- The nodes close to the surface are denoted by D1.

- $\quad \mathrm{D} 2$ represents nodes that remain in the water at a depth of level 2.

- $\quad$ Nodes near the seabed are designated as D3.

where D3 > D2 > D1 > D0

- Each layer contains several clusters, each with a cluster head and a cluster member. For example, the cluster head at depth D1 is node S3, and the cluster head at depth D2 is node S9.

- Nodes at the intersection of two layers will act as cluster relays, forwarding data from the deeper layer to the sink. Node S5, for example, becomes a cluster relay, forwarding data from cluster S9 to cluster S3 and then transmitting to the sink node.

- The multi-hop routing route to the sink is taken by node $\mathrm{S} 13$, which is situated on the seabed, through clusters of S12-S9-S5-S3-S1-sink.

This paper proposes a Managed Transmission Power-based Sparsity-Aware EnergyEfficient Clustering (CTP-SEEC) routing protocol for UWSNs in a spherical and square areas that is energy efficient and uses minimal transmission power. According to the identified upper and lower threshold values, it adjusts transmission power to a suitable Transmission Power Level (TPL). A sender node, for example, uses its modified Transmission Power Level (TPL) stored in the routing table to transmit data to its neighboring node. Receiving acknowledgment with the Received Signal Strength Indicator (RSSI) value would cause 
the sender node to change its TPL adaptively. The condition between higher and lower threshold values is used by the sender. It contrasts the RSSI value received with the lower and upper RSSI threshold values. The sender node tunes its TPL accordingly if it crosses one of these values, converting to a suitable power level. This is often done to reduce energy consumption by overcoming time-varying acoustic channel quality. It may, however, minimize network connectivity, increase the number of hops, and increase the end-to-end delay. Each node is given the appropriate transmitting power. The suggested routing scheme employs adaptive power control, which converts to a suitable Transmission Power Level (TPL) based on specified upper and lower threshold values, which is better than previous ones by using maximum energy in different sensor scenarios, the minimum transmission power used to increase overall network lifetime, packet delivery ratio, and interference. Using an adaptive communication power control mechanism, the proposed routing protocol is used to eliminate channel interference while also reducing energy consumption in sparse areas. Furthermore, the dense regions are divided into clusters to decrease repetitive transmissions as shown in Figure 1. By gathering data packets at a nearby header node, this will help to mitigate interference. A composite data packet is sent to the endpoint by this node. Based on the maximum residual power, Cluster Heads are chosen. $\mathrm{CHs}$ link to both the sink node with $\mathrm{CH}$ neighbors and the forward data packets, while handheld sinks are in dispersed areas to collect minimum energy consumption information, as shown in Figure 1. Sparse and denser regions are determined through the algorithms followed by the SEEC protocol, based on the density of the nodes in each logical area, the sparse and dense areas are specified. For example [7], assumed to be picked as a dense area, there should be $\mathrm{N}$ number of nodes to satisfy the limited threshold by using the following equation:

$$
N_{t h}= \begin{cases}1 & \text { if } N \geq N_{t h} \\ 0 & \text { if } N<N_{t h}\end{cases}
$$

The area is dense, when $\mathrm{N}$ is greater or equal to the $\mathrm{Nth}$ and the opposite indicates a sparse area. The proposed scheme incorporates the mechanism of transmission power control strategy. Two algorithms are suggested, which is most appropriate for comparison with our proposed algorithm [8]: (1) Sparsity Search Algorithm (SSA) to find sparse regions and (2) Density Search Algorithm (DSA) to find dense regions in the network place. In addition, the cluster head nodes automatically decided by the network and dynamically according to 3 conditions are met [8]:

- If the node's depth is lower than the other nodes of the density region in the present round

- Where Rest Power of the cluster head $(\mathrm{PCH})$ is greater than the average rest power calculated for an individual node.

- The node can be the cluster head if it has not been cluster head for the last $1 / \mathrm{p}$ rounds, the probability of a node to be a cluster head and it is calculated at the current round denoting the threshold (Th), the node generates a random number and, the compares the rand value with Th. The node can be CH If rand of the node is less than Th value, and only one $\mathrm{CH}$ node is specified in each density.

It consists of two phases: (i) Setup phase and (ii) Communication phase.

\subsection{Setup Phase}

In this paper, the sensor field is distributed into $\mathrm{n}$ sections, and relay nodes are arbitrarily positioned in the particular network area as shown in Figure 1. There is one fixed sink at the surface area and mobile sinks are positioned inside the sparse region. A ping message (control packet) is advertised by sink nodes across the network. This message has all the related facts of the sink node. Receiving nodes get the ping message from the fixed Sink and update its distance, when the routing path is setup, in the form of the number of hops from the sink fixed at the surface. Moreover, the receiving node sets a suitable Transmission Power Level (TPL) with the sink based upon the Received Signal Strength Indicator (RSSI). Along these lines, each node gets its distance from the 
sink, sets a suitable TPL, and then broadcasts this information in its transmission range. The same procedure is followed by other nodes. If a node receives a value greater than what it already has, it disposes of the ping message otherwise, it refreshes its distance information, sets a suitable TPL, and again broadcasts the ping message to nodes in its transmission range. This procedure proceeds till each sensor node sets a suitable TPL with the selected neighbors and obtains its distance from the sink. In short, control packets in the form of ping message contains a cost field which reflects the number of hops to reach the sink. In the setup phase, such control packets are propagated throughout the network. This results in establishing a minimum or shortest route from source to sink along with TPL adjustment of each node with its selected neighboring nodes. The parameters of simulation are shown in Table 1.

Table 1. Simulation Parameters Used for CTP-SEEC Performance Evaluation.

\begin{tabular}{cc}
\hline Simulation Parameters & Values \\
\hline Node numbers & 100 \\
Nodes' initial energy & $5 \mathrm{~J}$ \\
Data rate & $16 \mathrm{Kbps}$ \\
Band width & $16 \mathrm{Kbps}$ \\
Center frequency & $30 \mathrm{kHz}$ \\
Size of packet & $50 \mathrm{Bytes}$ \\
Reception power packet & $0.1 \mathrm{~W}$ \\
Transmission power packet & $2 \mathrm{~W}$ \\
Range of transmission & $50 \mathrm{~m}$ \\
Running rounds & 3500 \\
\hline
\end{tabular}

\subsection{Data Communication Phase}

By gathering data packets at a nearby header node, this will help to mitigate interference. A composite data packet is sent by this node to the endpoint. Based on maximum residual power, $\mathrm{CHs}$ are chosen. $\mathrm{CHs}$ connect to both the sink node or with $\mathrm{CH}$ neighbors and the forward data packets, while handheld sinks are in dispersed areas to gather data with minimal energy consumption. If the RSSI reaches its predefined limit stated as either low or high, the sender node tunes its TPL accordingly, i.e., if the RSSI is below the limit, it switches to the next power level; on the other hand, if the RSSI is above the high limit, the power level will degrade to a level. The RSSI limits are fixed at the start of the data communication phase and it depends on the channel condition. The process of the proposed technique is depicted in Figure 3, where $\mathrm{n}$ is the number of nodes, $\mathrm{R}$ is the region, $\mathrm{r}$ is the rGR which is donated searching the number of sparsity and density regions of the network. 


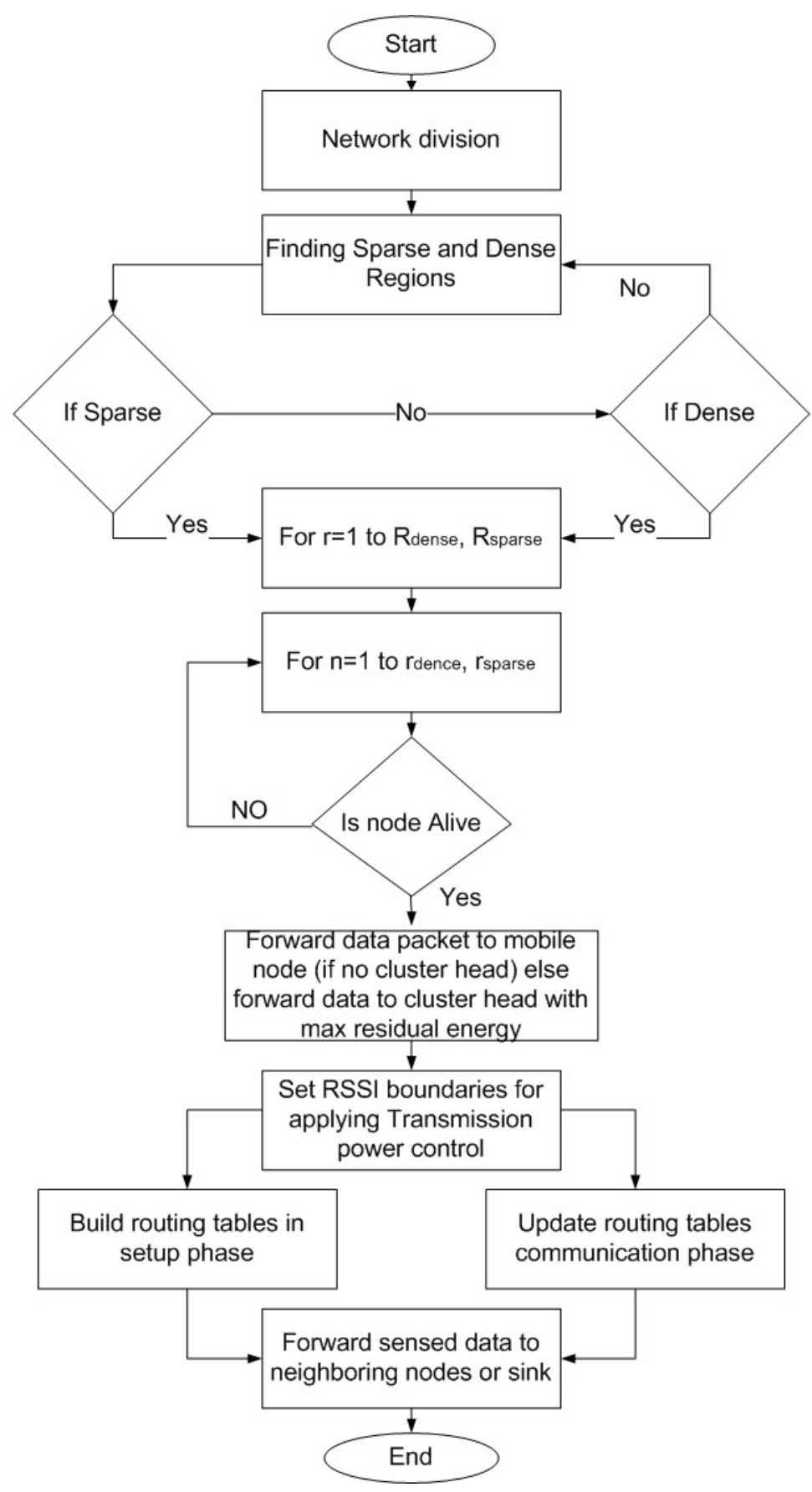

Figure 3. Network Flow Chart.

\section{Simulation Results and Discussions}

This section explains the estimation of the efficiency of the proposed method in terms of the life of the network, the stability period, the residual energy, the packets received per round and the total packets received at the sink. To verify the new system, the latest stateof-the-art schemes are extensively analyzed. The underwater routing schemes DBR [10], EEDBR [30], and SEEC [9] were taken into account for this reason. We have modeled these protocols by using Matlab 2017b/Simulink software, and the simulation cycle consists of 3500 rounds with the same set of environmental parameters to provide reliable performance. In a $100 \mathrm{~m} \times 100 \mathrm{~m}$ underwater area, the total number of 100 sensor nodes is deployed randomly. Initially, each node contains 5 joules of energy. Each sensor node can transmit in the range of $50 \mathrm{~m}$. The depth threshold is set to $15 \mathrm{~m}$. The proposed protocol is evaluated based on the following metrics: 
The proposed scheme is evaluated considering the following standard evaluation metrics:

- Network Lifetime: These metrics indicate how long the network nodes operate. This refers to the cumulative number of rounds when the network field's nodes are both alive and working. To consider the efficacy of a system, this metric is essential as energy is the primary concern of UWSNs. In the underwater world, the aim of most mechanisms is to prolong the lifespan of the network. Basically, it depends on energy consumption.

- Throughput: Throughput is called the efficient reception of the data packet at the sink. Data transmission to the sink depends on the number of nodes being involved. More active nodes contribute sufficient packets to the sink, which increases the network throughput.

- $\quad$ Packets received per round: This review shows the ratio and the capacity of packets received at the sink in the unique round to accept the data packets. If the ratio exceeds the threshold for sending packets, the packet will be dropped. Residual Energy: Residual Energy is the difference between the initial energy and total energy consumed by each node after data packets have been transmitted and received.

\subsection{Network Lifetime}

After introducing the managed transmission power function in SEEC, as seen in Figure 4, the network lifetime is enhanced. In SEEC, each sensor node was transmitting packets at a predefined transmission power which made the network die prematurely. In the controlled transmission power (CTP) strategy, transmission power is adjusted by the source node based on Euclidean distance to the destination node. These adjustments of power vary energy consumption for each time when the packet is sent. This variation in energy consumption increases the network lifetime. In Figure 4, it can be seen that the nodes in SEEC with CTP are alive at round 3500 whereas the SEEC without CTP is dead at round 2500 as shown in Figure 5. In DBR During the information sending, the sender incorporates its depth; the data packets accepting nodes contrast their depths with the sender node. The node with a small depth advances the packet to the sink. For each data packet, each node has a fixed holding time, so nodes with greater depths have a smaller holding time. Therefore, because only the depth of the sensor node is used for propagation, these nodes die before alternative nodes that lead to energy holes; see Figure 5. The nodes in DBR with CTP are alive at round 3500, whereas the DBR without CTP is dead at round 2000, as seen in Figure 4.

In EEDBR, the stability duration is better than DBR, as seen in Figure 4; and when the network is sparse, node neighbors quickly decrease the network's instability and the node load is also higher in EEDBR, so the number of dead nodes in Figure 5 increases suddenly. It can be shown in Figure 4 that the EEDBR nodes with CTP are alive at round 3500, whereas the EEDBR without CTP is dead at round 2000, as seen in Figure 5. 


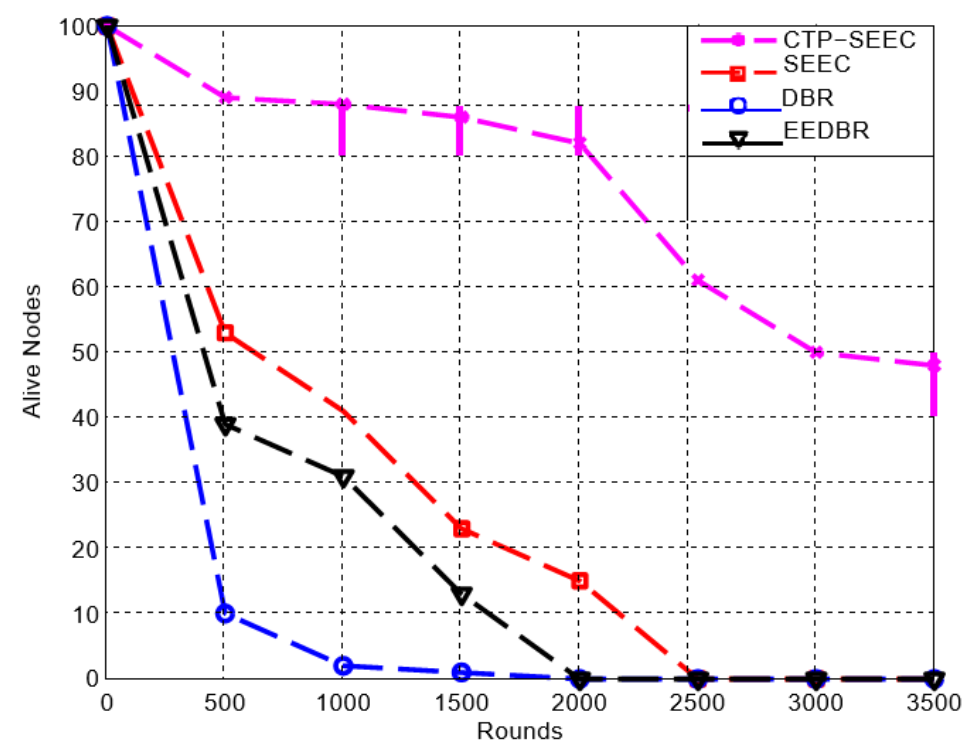

Figure 4. Network Lifetime.

\subsection{Stability Period}

Sensor node random deployment does not necessarily give the same number of dense and sparse regions. If the number of dense regions is maximal and the nodes interact with the same transmission power within the same dense regions, the stability duration is compromised.

On the other hand, the regulated transmission power technique is implemented, as seen in Figures 4 and 5, as a result the stability period is increased. In addition to regulated transmission capacity, the clustering of denser regions used and the relocation of mobile sink nodes in sparse regions in the CPT-SEEC enhances the stability period relative to SEEC, EEDBR and DBR. In DBR, the greedy process forces immutable forwarder node selection when network nodes are partitioned and resources remain underused, allowing the protocol to have the lowest stability duration. It can be seen that the nodes in DBR without CTP are dead in round 2000 as shown in Figure 5. In EEDBR, because of excessive data forwarding and rapid energy reduction, nodes start to die quickly.

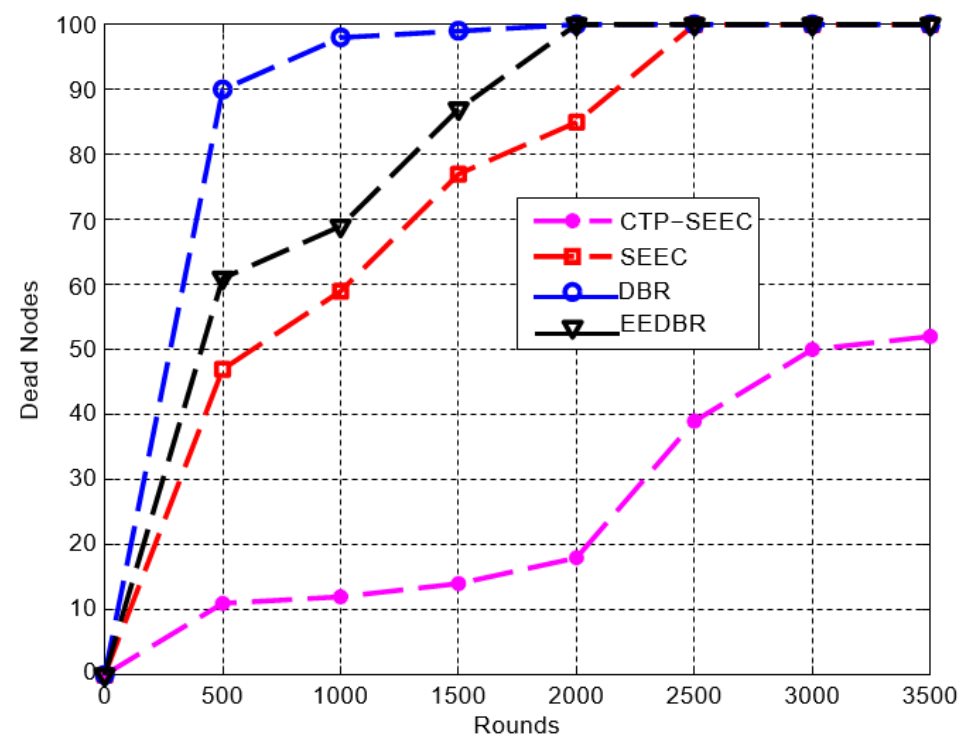

Figure 5. Stability Period. 


\subsection{Saturation Throughput}

Figure 6 shows the behavior of saturation throughput growth of network during simulation rounds, and suggests that the cumulative packets collected at the sink are higher in CTP-SEEC. This is attributed to the improved lifespan of the network with the capacity policy of controlled transmission. In addition to this, CTP-SEEC's expanded throughput also results in a high number of live nodes during the network lifespan. Packets obtained per round in the EEDBR result in high residual energy, while a high packet distribution ratio in the sparse area cannot be accomplished by choosing nodes and by considering residual energy. In DBR, only the low-depth node is considered for packet routing, which often results in packet replication, allowing DBR to reach the lowest throughput compared to other protocols. Under the SEEC protocol, the number of packets received at the sink node is less than the suggested CTP-SSEC routing protocol. The theory behind this is that the transmitting power stays steady in SEEC and the network dies early. Figure 7 shows that the packets received per round with controlled transmission power are better than SEEC.

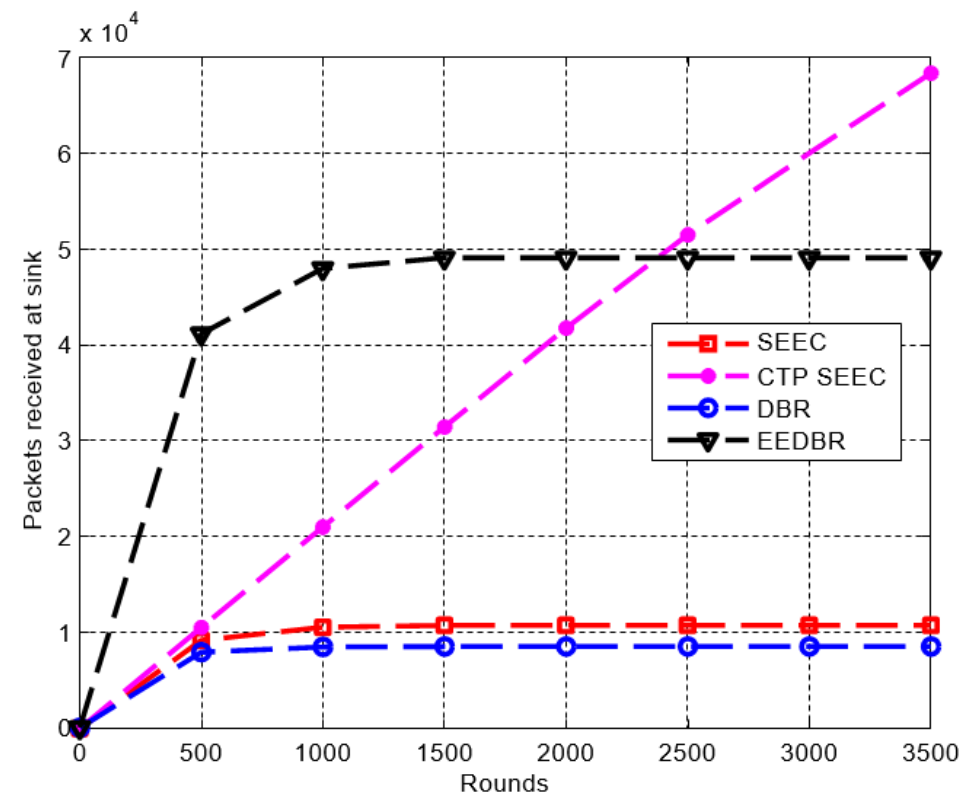

Figure 6. Saturation throughput.

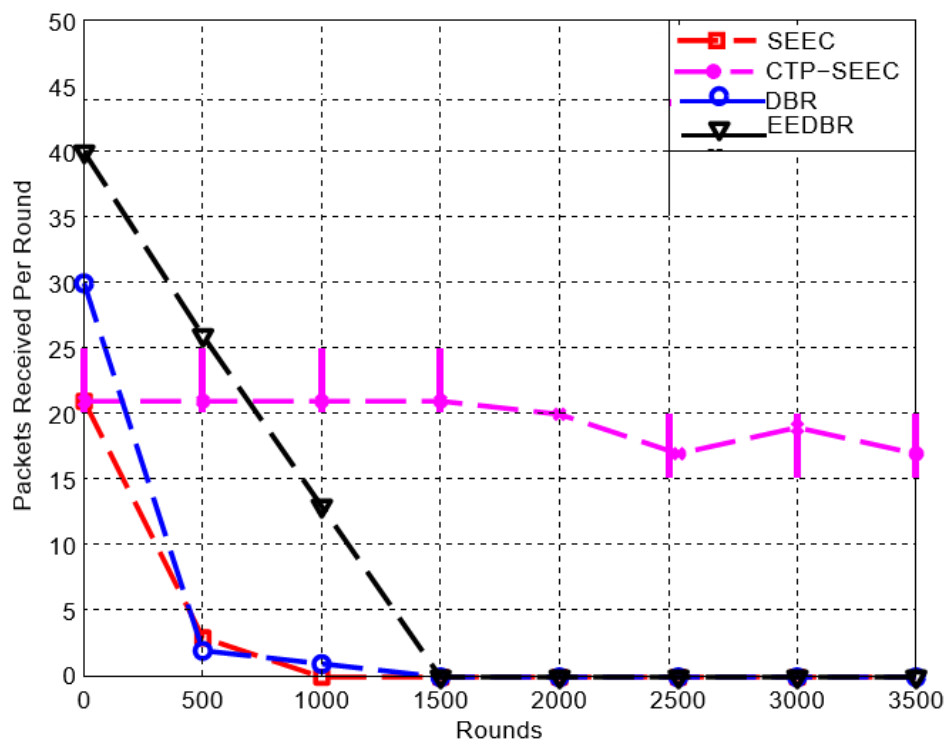

Figure 7. Packets Received Per Round. 


\subsection{Residual Energy}

In SEEC, the factor of transmission power in the energy consumption was constant. Regardless of the distance between two communicating nodes, almost the same amount of energy we are consuming while transmitting a packet. In CTP-SEEC, the function of energy consumption varies.

After sending the packet, the return value contributes to higher network residual capacity. Transmission power is balanced in the suggested scheme according to the distance between the source and the destination node.

The less the distance, the less the transmission power, and vice versa. Less transmission power results in less energy tax which eventually results in higher network residual energy in CTP-SEEC. DBR considers only the depth; it does not consider the residual energy, so it has the minimum residual energy. In EEDBR, a high packet distribution ratio in the sparse area cannot be accomplished by choosing nodes and by considering residual capacity. In Figure 8, the cumulative network residual energy consumption of CPT-SEEC, SEEC, EEDBR, and DBR is observed.

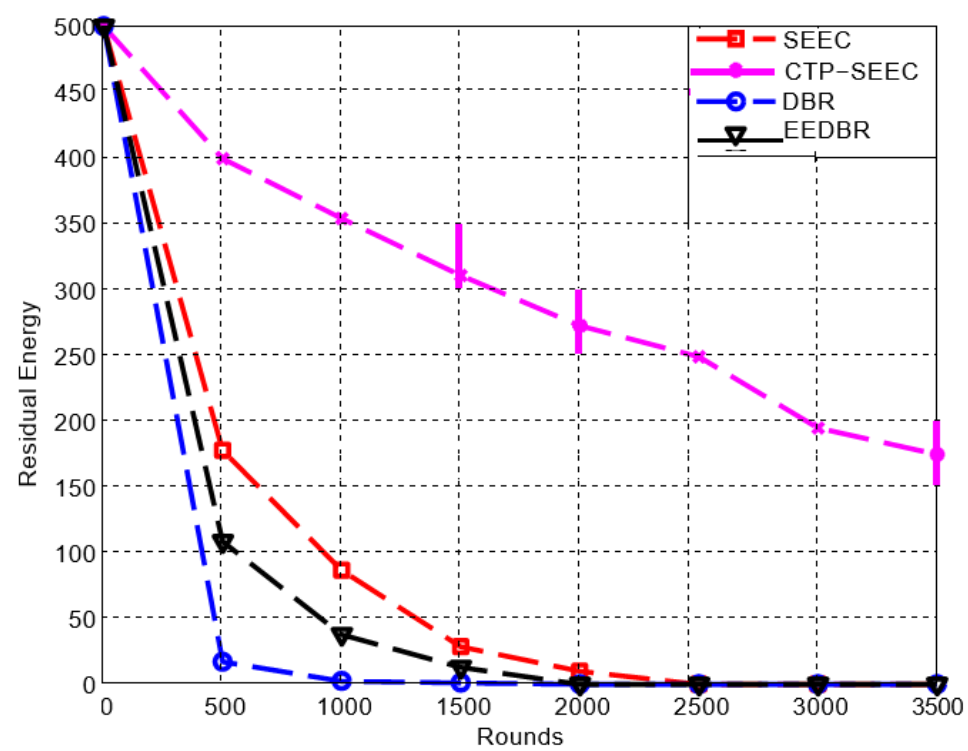

Figure 8. Residual Energy.

\subsection{Simulation Variable Environment}

Our simulation environment consists of dynamic nodes deployed sparsely in a 3D environment $(500 \mathrm{~m} \times 500 \mathrm{~m} \times 500 \mathrm{~m})$. Herein, we run the simulation cycle for 1000 rounds. Moreover, to check the scalability of the proposed work, we change the number of nodes and their transmission ranges varying from 50 to 250 and $15 \mathrm{~m}$ to $200 \mathrm{~m}$, respectively as in [34].

The proposed protocol is evaluated based on residual energy and packets received that will be impacted on the network lifetime and throughput, respectively. In terms of residual energy metric, the Figure 8 shows three protocols reduced the residual energy. However, the residual energy in CTP-SEEC is higher than in SEEC, or DBR, during a varied number of sensor nodes. In DBR, the increase in the number of nodes in the network shows that more data packets can be received by the forwarding node. In turn, it sends these packets to the upper level before reaching the sink. Here, DBR elects the upper-level depth to be its forwarder node, and this process results in high energy consumption due to high transmission at the forwarders node. In SEEC, the protocol performs better than the DBR in the case of a low transmission range which ensures a long network lifetime. The node that selects to be a $\mathrm{CH}$ based on its depth and residual energy makes all the nodes efficient to save energy in case of a varying number of nodes and transmission range. However, the cluster head broadcasts information to the three-dimensional area network, the nodes 
consume energy gradually. However, CTP-SEEC can be performed as a CH or CR, thus the cluster head has the information of its cluster members, which reduces the number of transmissions between nodes.

Figure 9 shows the higher energy consumption in CTP-SEEC, SEEC, and DBR, when decreasing the transmission range. In CTP-SEEC, the residual energy within a $200 \mathrm{~m}$ transmission range is more than a $150 \mathrm{~m}$ transmission range. The cluster heads collect the data of all the cluster members and then forward these to the cluster relay which belongs to another cluster.

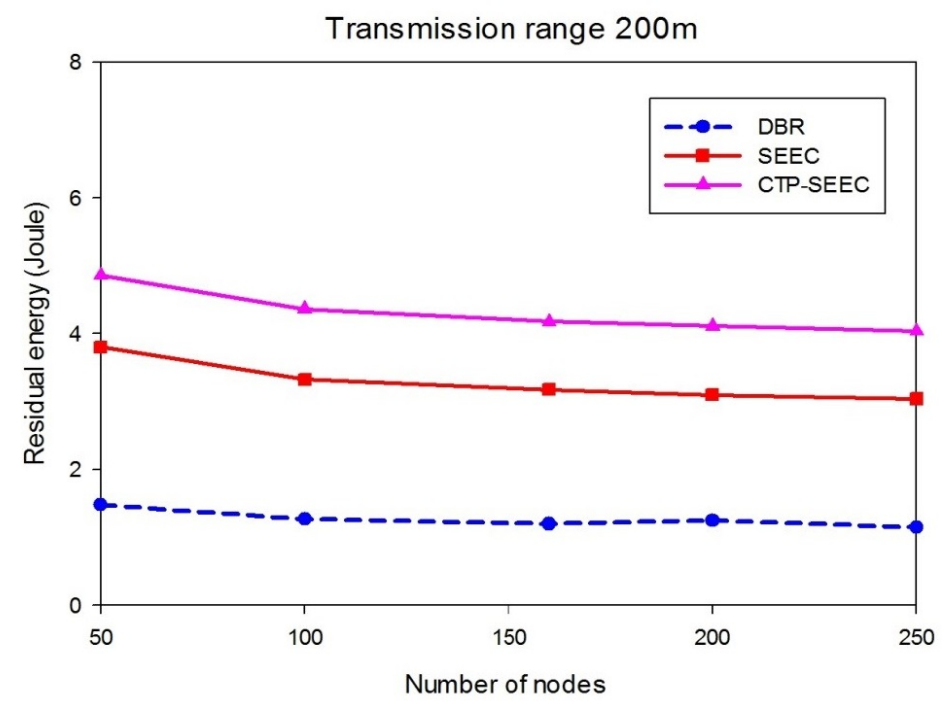

Figure 9. Residual energy: Transmission range $200 \mathrm{~m}$, nodes from 50 to 250.

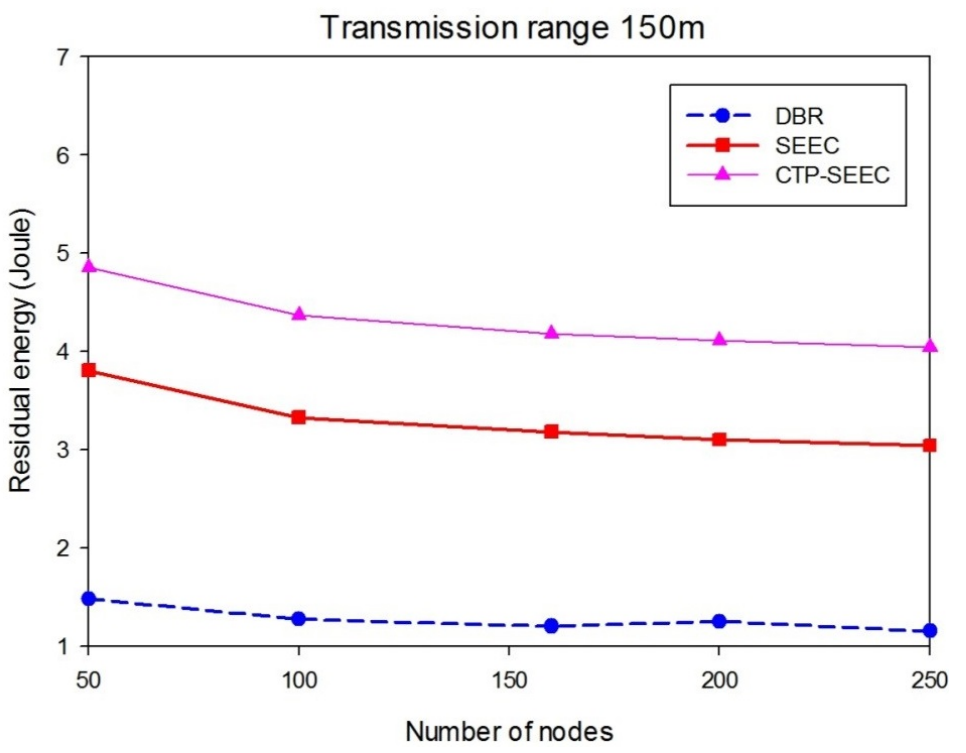

Figure 10. Residual energy: Transmission range $150 \mathrm{~m}$, nodes from 50 to 250.

In terms of received packets metrics, the network topology changes every 1000 rounds during the deployment, which causes an error in received packets at cluster head and cluster relay because the distance from the cluster member to the cluster head greater than the transmission range that makes fail received packets at the cluster head or cluster members leads to reduce the level of received power. Thus, the cluster should be a renewed setup, a cluster head, a cluster member, and a cluster relay must be re-elected. Figure 10 shows lower residual energy at the node during the increased transmission range. 
In Figure 11, we can see that by changing the transmission range to $200 \mathrm{~m}$, the received packets at the sink increases in CTP-SEEC protocol, which is higher than in SEEC, or DBR. It increases the received packets at the sink with increase to the transmission range and it is periodically different from the total number of received packets in CTP-SEEC.

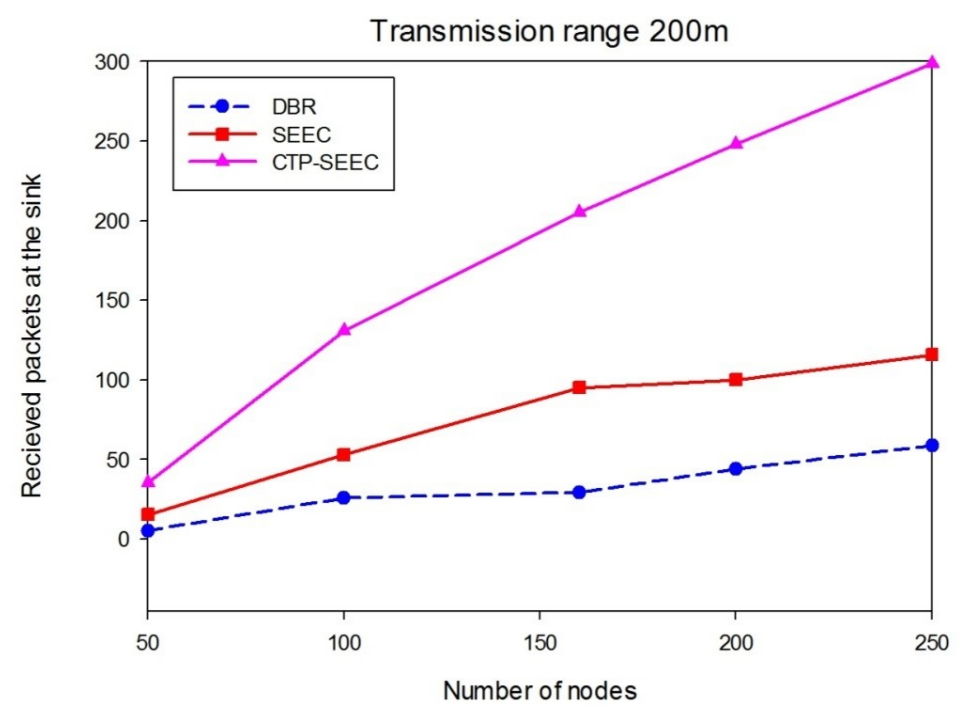

Figure 11. Received packets at the sink: Transmission range $200 \mathrm{~m}$, nodes from 50 to 250.

Figure 12 shows that the received packets at the sinks in CTP-SEEC are higher than other protocols, which results in high throughput and a more reliable network.

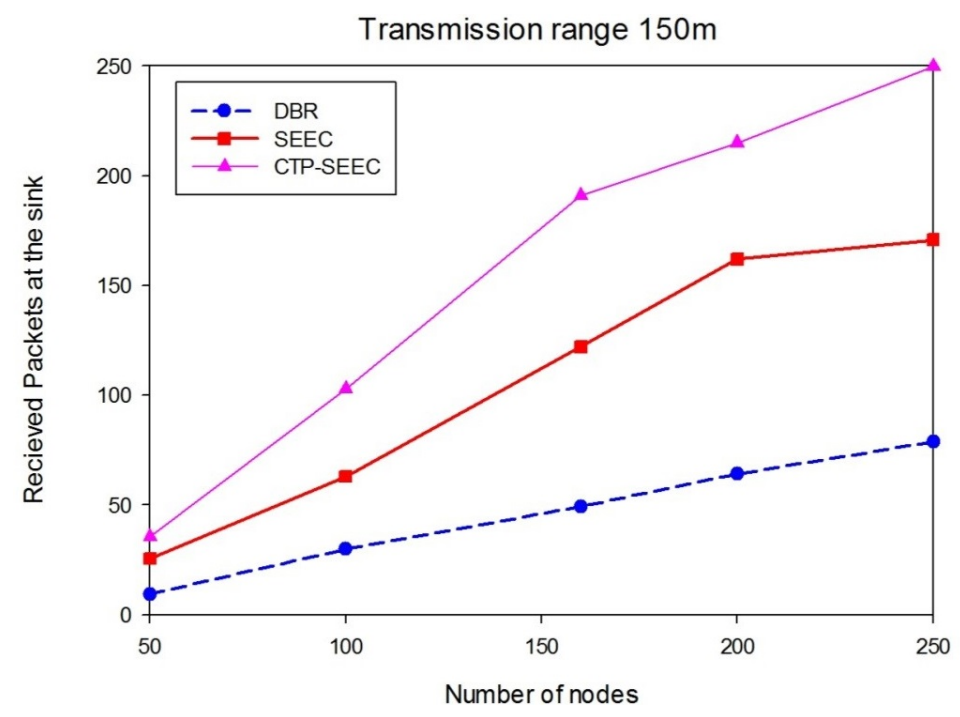

Figure 12. Received packets at the sink: Transmission range $150 \mathrm{~m}$, nodes from 50 to 250 .

\section{Conclusions}

The energy hole creation, high interference, unbalanced data load distribution, and naive node-level energy consumption approaches are considered some of the key reasons for poor UWSN performance. The integration of various compatible approaches often results in improved network performance. This paper incorporates three state-of-the-art UWSN approaches: transmit power control, mobile sink use, and clustering. The results of the assessment validate the fact that the ratio of network life, throughput, and packet distribution can be maximized by integrating the regulation of node-level transmission power, clustering in dense regions, and the use of mobile sinks in sparse network regions. The formation of energy holes is considered one of UWSN's most important problems. 
The proposed scheme has addressed this problem efficiently; however, it is observed that the smart and adaptive mobility mechanism of mobile sinks has a great impact on the avoidance of energy hole creation.

Author Contributions: The research conceptualization and methodology are done by I.A.H., S.N.K. and F.A. (Faisal Althobiani). The technical and simulations are performed by M.I. (Muhammad Irfan), M.I. (Muhammad Idrees) and S.U. The technical review and improvements have been performed by F.A. (Faisal Alsaaqq), A.G., I.G., R.M. and M.T. The overall technical support, guidance and project administration is done by R.T. and M.T. All authors have read and agreed to the published version of the manuscript.

Funding: This research was funded by the EMMAT E-mobility and sustainable materials and technologies (E-mobilność oraz zrównoważone materiały i technologie) Projekt nr PPI/APM/2018/1/00027.

Acknowledgments: The authors acknowledge the support from the Najran University, Kingdom of Saudi Arabia.

Conflicts of Interest: There is no conflict of interest.

\section{Abbreviations}

$\begin{array}{ll}\text { AUV } & \text { Autonomous Underwater Vehicle } \\ \text { Co-LFEER } & \text { Localization-Free Energy-Efficient Cooperative Routing } \\ \text { CTP } & \text { Controlled Transmission Power } \\ \text { CTP-SEEC } & \text { Controlled Transmission Power-Based Sparsity-Aware Energy Efficient Clustering } \\ \text { LFEER } & \text { Localization-Free Energy-Efficient Routing } \\ \text { LQI } & \text { Link Quality Indication } \\ \text { MUR } & \text { Mobile Sink Utility Ratio } \\ \text { PDR } & \text { Packet Delivery Ratio } \\ \text { PRP } & \text { Packet Reception Probability } \\ \text { QoS } & \text { Quality of Service } \\ \text { RSSI } & \text { Received Signal Strength Indicator } \\ \text { SNR } & \text { Signal-to-Noise Ratio } \\ \text { TPL } & \text { Transmission Power Level } \\ \text { TWSNs } & \text { Terrestrial Wireless Sensor Networks } \\ \text { UWSN } & \text { Underwater Wireless Sensor Networks } \\ \text { WSN } & \text { Wireless Sensor Network }\end{array}$

\section{References}

1. Yu, H.; Yao, N.; Wang, T.; Li, G.; Gao, Z.; Tan, G. Ad Hoc Networks WDFAD-DBR: Weighting depth and forwarding area division DBR routing protocol for UASNs. Ad Hoc Netw. 2016, 37, 256-282. [CrossRef]

2. Yu, H.; Yao, N.; Liu, J. Ad Hoc Networks An adaptive routing protocol in underwater sparse acoustic sensor networks. Ad Hoc Netw. 2015, 34, 121-143. [CrossRef]

3. Parra, L.; Sendra, S.; García, L.; Lloret, J. Design and Deployment of Low-Cost Sensors for Monitoring the Water Quality and Fish Behavior in Aquaculture Tanks during the Feeding Process. Sensors 2018, 18, 750. [CrossRef] [PubMed]

4. Sendra, S.; Lloret, J.; Jimenez, J.M.; Parra, L. Underwater acoustic modems. IEEE Sens. J. 2016, 16, 4063-4071. [CrossRef]

5. Han, G.; Shu, L.; Rodrigues, J.J.; Kim, K.; Lloret, J.; Wu, H. Guest Editorial Special Issue on Advances in Underwater Acoustic Sensor Networks. IEEE Sens. J. 2016, 16, 3994. [CrossRef]

6. Coutinho, R.W.L.; Boukerche, A.; Vieira, L.F.M.; Loureiro, A.A.F. Geographic and OpportunisticRouting for Underwater Sensor Networks. IEEE Trans. Comput. 2016, 65, 548-561. [CrossRef]

7. Sher, A.; Javaid, N.; Azam, I.; Ahmad, H.; Abdul, W.; Ghouzali, S.; Niaz, I.A.; Khan, F.A. Monitoring square and circular fields with sensors using energy-efficient cluster-based routing for underwater wireless sensor networks. Int. J. Distrib. Sens. Netw. 2017, 13, 1550147717717189. [CrossRef]

8. Azam, I.; Majid, A.; Ahmad, I.; Shakeel, U.; Maqsood, H.; Khan, Z.A.; Qasim, U.; Javaid, N. SEEC: Sparsity-aware energy-efficient clustering protocol for underwater wireless sensor networks. In Proceedings of the 2016 IEEE 30th International Conference on Advanced Information Networking and Applications (AINA), Crans-Montana, Switzerland, 23-25 March 2016; pp. 352-361.

9. Ahmed, G.; Khan, N.M. Adaptive power-control based energy-efficient routing in wireless sensor networks. Wirel. Pers. Commun. 2017, 94, 1297-1329. [CrossRef]

10. Yan, H.; Shi, Z.J.; Cui, J.H. DBR: Depth-based Routing for Underwater Sensor Networks. In International Conference on Research in Networking; Springer: Berlin/Heidelberg, Germany, 2008; pp. 72-86. 
11. Pari, S.; Neelavathy, M.; Sathish, K. Arumugam. An energy-efficient and reliable depth-based routing protocol for underwater wireless sensor network (ER-DBR). In Advances in Power Systems and Energy Management; Springer: Singapore, 2018 ; pp. 451-463.

12. Yahya, A.; Islam, S.U.; Akhunzada, A.; Ahmed, G.; Shamshirband, S.; Lloret, J. Towards Efficient Sink Mobility in Underwater Wireless Sensor Networks. Energies 2018, 11, 1471. [CrossRef]

13. Shah, S.; Khan, A.; Ali, I.; Ko, K.M.; Mahmood, H. Localization Free Energy Efficient and Cooperative Routing Protocols for Underwater Wireless Sensor Networks. Symmetry 2018, 10, 498. [CrossRef]

14. Kong, L.; Ma, K.; Qiao, B.; Guo, X. Adaptive relay chain routing with load balancing and high energy efficiency. IEEE Sens. J. 2016, 16, 5826-5836. [CrossRef]

15. Zhou, Z.; Yao, B.; Xing, R.; Shu, L.; Bu, S. E-CARP: An energy efficient routing protocol for UWSNs in the internet of underwater things. IEEE Sens. J. 2015, 16, 4072-4082. [CrossRef]

16. Noh, Y.; Lee, U.; Lee, S.; Wang, P.; Vieira, L.F.; Cui, J.H.; Gerla, M.; Kim, K. Hydrocast: Pressure routing for underwater sensor networks. IEEE Trans. Veh. Technol. 2016, 65, 333-347. [CrossRef]

17. So, J.; Byun, H. Load-balanced opportunistic routing for duty-cycled wireless sensor networks. IEEE Trans. Mob. Comput. 2017, 16, 1940-1955. [CrossRef]

18. Karim, S.; Shaikh, F.K.; Chowdhry, B.S.; Mehmood, Z.; Tariq, U.; Naqvi, R.A. Ahmed, A; GCORP: Geographic and cooperative opportunistic routing protocol for underwater sensor networks. IEEE Access 2021, 9, 27650-27667. [CrossRef]

19. Sahana, S.; Singh, K.; Kumar, R.; Das, S. A review of underwater wireless sensor network routing protocols and challenges. Next Gener. Netw. 2018, 638, 505-512.

20. Abbas, M.Z.; Bakar, K.A.; Ayaz, M.; Mohamed, M.H.; Tariq, M. Hop-by-Hop Dynamic Addressing Based Routing Protocol for Monitoring of long range Underwater Pipeline. KSII Trans. Internet Inf. Syst. 2017, 11, 731-759.

21. Khan, A.; Ali, I.; Rahman, A.U.; Imran, M.; Mahmood, H. Co-EEORS: Cooperative Energy Efficient Optimal Relay Selection Protocol for Underwater Wireless Sensor Networks. IEEE Access 2018, 6, 28777-28789. [CrossRef]

22. Yadav, S.; Kumar, V. Optimal Clustering in Underwater Wireless Sensor Networks: Acoustic, EM and FSO Communication Compliant Technique. IEEE Access 2017, 5, 12761-12776. [CrossRef]

23. Sasikala, V.; Chandrasekar, C. Cluster based Sleep/Wakeup Scheduling Technique for WSN. Int. J. Comput. Appl. 2013, 72, 15-19.

24. Yu, S.; Liu, S.; Jiang, P. A High-Efficiency Uneven luster Deployment Algorithm Based on Network Layered for Event Coverage in UWSNs. Sensors 2016, 16, 2103. [CrossRef]

25. Rao, P.S.; Jana, P.K.; Banka, H. A particle swarm optimization based energy efficient cluster head selection algorithm for wireless sensor networks. Wirel. Netw. 2017, 23, 2005-2020. [CrossRef]

26. Ez-Zazi, I.; Arioua, M.; El Oualkadi, A.; Lorenz, P. On the performance of adaptive coding schemes for energy efficient and reliable clustered wireless sensor networks. Ad Hoc Netw. 2017, 64, 99-111. [CrossRef]

27. Sher, A.; Khan, A.; Javaid, N.; Ahmed, S.H.; Aalsalem, M.Y.; Khan, W.Z. Void Hole Avoidance for Reliable Data Delivery in IoT Enabled Underwater Wireless Sensor Networks. Sensors 2018, 18, 3271. [CrossRef] [PubMed]

28. Mahmood, S.; Nasir, H.; Tariq, S.; Ashraf, H.; Pervaiz, M.; Khan, Z.A.; Javaid, N. Forwarding nodes constraint based DBR (CDBR) and EEDBR (CEEDBR) in underwater WSNs. Procedia Comput. Sci. 2014, 34, 228-235. [CrossRef]

29. Javaid, N.; Ejaz, M.; Abdul, W.; Alamri, A.; Almogren, A.; Niaz, I.; Guizani, N. Cooperative Position Aware Mobility Pattern of AUVs for Avoiding Void Zones in Underwater WSNs. Sensors 2017, 17, 580. [CrossRef] [PubMed]

30. Islam, T.; Lee, Y.K. A Comprehensive Survey of Recent Routing Protocols for Underwater Acoustic Sensor Networks. Sensors 2019, 19, 4256. [CrossRef]

31. Sheng, M.; Tang, S.; Qin, H.; Wan, L. Clustering Cloud-Like Model-Based Targets Underwater Tracking for AUVs. Sensors 2019, 19, 370. [CrossRef]

32. Patil, K.; Jafri, M.; Fiems, D.; Marin, A. Stochastic modeling of depth based routing in underwater sensor networks. Ad Hoc Netw. 2019, 89, 132-141. [CrossRef]

33. Coutinho, R.W.L.; Boukerche, A.; Vieira, L.F.M.; Loureiro, A.A.F. Local Maximum Routing Recovery in Underwater Sensor Networks: Performance and Trade-offs. In Proceedings of the 2014 IEEE 22nd International Symposium on Modelling, Analysis \& Simulation of Computer and Telecommunication Systems, Washington, DC, USA, 9-11 September 2014; pp. 112-119.

34. Nguyen, N.T.; Le, T.T.; Nguyen, H.H.; Voznak, M. Energy-efficient clustering multi-hop routing protocol in a UWSN. Sensors 2021, 21, 627. [CrossRef] 